\title{
Téoros
}

Revue de recherche en tourisme

\section{Cap sur le Saint-Laurent, un témoignage}

\section{Benoît Gauthier}

Volume 6, numéro 2, juillet 1987

Le Saint-Laurent magnétique

URI : https://id.erudit.org/iderudit/1080510ar

DOI : https://doi.org/10.7202/1080510ar

Aller au sommaire du numéro

Éditeur(s)

Université du Québec à Montréal

ISSN

0712-8657 (imprimé)

1923-2705 (numérique)

Découvrir la revue

Citer ce document

Gauthier, B. (1987). Cap sur le Saint-Laurent, un témoignage. Téoros, 6(2),

30-32. https://doi.org/10.7202/1080510ar d'utilisation que vous pouvez consulter en ligne.

https://apropos.erudit.org/fr/usagers/politique-dutilisation/ 


\title{
Cap sur le Saint-Laurent un témoignage
}

\author{
par Benoît Gauthier *
}

\section{L'apprentissage du Saint-Laurent}

Mes premiers véritables contacts avee le Saint-Laurent ont commencé avec un projet de maítrise en écologie végétale à l'automne 1969: j'embarquais alors à Montmagny. Aprés deux étés passés successivement à Saint-Lén au Lac-Saint-Jean et à Poste-de-la-Baleine au NouveauQuébec, j'avais décidé de travailler dans la vallée du Saint-Laurent, là où la quasitotalité des Québécois vivent, aménagent, développent et se recréent.

En 1969, je devins également administrateur de la Société linnéenne de Québec. C'est là que, petit à petit, je devais apprendre à côtoyer des chercheurs, des éducateurs, de bons vulgarisateurs et surtout a communiquer au public le résultat de mes découvertes et connaissances.

Par la suite, mes études doctorales sur l'ensemble du littoral du fleuve ainsi que des stages en 1972-1973 un stage en Nouvellè-Zelande et, en 1974, à Montpellier pour en étudier notamment les marais vont contribuer à me convaincre que le Saint-Laurent offre un modèle unique dans le monde. Il est un autre enseignement que je retiens de toutes ces années de terrain: la pollution ne peut être corrigée sans changer profondément l'attitude des urbains à la source, $c^{*}$ est-ă-dire dans le milieu même où ils vivent.

Sept années d'études le long du SaintLaurent s'achèvent. Alors, l'occasion se présente de travailler comme conseiller scientifique au Conseil consultatif de l'environnement du Québec. J'ai le choix de poursuivre ma carrière en écologie végétale sur le Saint-Laurent et les divers milieux humides du Québec, tout en espérant que les pressions environnementales sur ces habitats vont diminuer, ou de m'engager là où l'on propose des choix sociaux pour le Québec. Je décide d'oeuvrer dans l'antichambre des décideurs, tout près des grands enjeux environnementaux

"Benoit Gauthier, est deologue et conseiller sciontifique au Conseil consututif de l'Emironnement du Cutboc. II fut bgalement pridsdent de le Socidte Linneenne du Ouébec. qui ont cours dans la vallée du SaintLaurent. J'accepte aussi de devenir viceprésident de la Société linnéenne, là où je peux laisser libre cours à mes idées et mettre en pratique des projets de sensibilisation du public aux sciences naturelles et à leur environnement urbain.

Le Conseil consultatif de l'environnement devait s'avérer un lieu intellectuel extraordinaire. La plupart des sujets d'importance sont abordés dont certains génèrent des avis au ministre de l'Environnement du Québec; certains concernent le Saint-Laurent: projet minéralo-portuaire aux Iles-de-laMadeleine, exploitation des algues marines dans le golfe du Saint-Laurent, rétrospective du mercure dans l'environnement québécois, les marais salés entre La Pocatière et l'Isle-Verte, politique de conservation du littoral, etc. Dans ces dossiers controversés où le public est informé et consulté sur ses préférences, $\mathrm{j}$ 'apprends qu'il faut avancer avec prudence et ne pas dépasser la capacité d'absorption de la population et ce, quelle que soit la légitimité des gestes.

\section{Le grand défi de la société linnéenne}

Le grand défi va survenir lorsque la Société linnéenne décide $d^{\prime}$ 'organiser une croisière aux baleines dans le cadre des activités de son cinquantenaire ${ }^{11}$. Nous disposons de peu d'informations sur l'endroit exact où vivent les baleines, sur la période de leur présence dans le Saint-Laurent, sur la logistique pour s'y rendre, sur la clientèle que cela intéresse, etc. Nous questionnons plusieurs personnes sans obtenir des renseignements fiables. Je me rends compte à nouveau jusqu'à quel point les Québécois ont tourné le dos au Saint-Laurent et aux ressources qu'il renferme. Un sondage auprès de nos trois cents membres révèlent que huit personnes seraient intéressées par une croisière de ce genre! Optimistes, nous multiplions ce nombre par cinq et nous dénichons une agence de voyage de Montréal, associée à la Zoological Society of Montreal, qui garantit les cent autres places sur le Gobelet d'argent, traversier privé de 55 mètres dont le port d'attache se trouve à Trois-Pistoles. C'était sans compter sur les principaux médias de Québec qui nous appuient dans notre publictés: en effet. 450 personnes réserveront en une seule semaine! La population francophone se réveille. Trois croisieres seront finalement nolisées entièrement par la Société linnéenne en août 1979 au coût de $50 \$$ pour 11 heures d'excursion. Au moins sept spécialistes de différentes facettes du SaintLaurent se portent bénévoles pour chacune de ces sorties bien spéciales. Les baleines sont au rendez-vous, c'est une véritable réussite.

La Sociêté linnéenne semble prête à mettre le cap sur le Saint-Laurent lorsque j"en accepte la présidence en octobre 1979. Les deux plus importants dossiers de cette première année seront la protection des marais de Kamouraska et la poursuite des croisières aux baleines. Nous y mettons toutes nos énergies à sensibiliser l"ensemble des Québecois sur ces deux ressources du SaintLaurent. Nos stratégies appuyées sur des dossiers étoffés donnent des résultats étonnants que nous mesurons quotidiennement par les encouragements que nous recevons de la population. L'année suivante, en 1981, nous arrivons à une entente avec Via Rail et les Musées nationaux, et le nombre de croisières passe de trois à onze; ce produit touristique s'étend dès lors à l'ensemble du Québec. Nous en venons à produire un diaporama, des films, macarons, souvenirs éducatifs, un livret sur les baleines et une affiche pour la protection des bélugas, la culture sur les baleines du SaintLaurent se répandant comme une trainée de poudre. Devant la popularité du produit et un taux de satisfaction supérieur a $95 \%$, nous contimuons de voir grand à l'été 1982 où pas moins de 27 croisières pour les baleines seront réalisées, dont trois à par= tir des Escoumins. La phase expérimentale èst achevée, et nous voilà entrés dans une phase commerciale avec la création d'une clientèle.

\section{La phase commerciale}

Stimulés par cette réussite et encouragés par notre partenaire Via Rail, nous mettons au point pour le printemps de la même année un produit axé sur l'omithologie, plus précisément sur l'observation des grandes oies blanches dans l'archipel de Montmagny: 31 sorties ont lieu à partir de Montmagny com- 
prenant un volet aquatique et un autre sur l'île aux Grues en minibus. Nous lançons à l'été (du 9 août au 6 septembre) les croisières intitulées "Entre la mer et l'eau douce", a partir cette fois du quai de Sainte-Anne-de-Beaupré. Le bilan de la Linnéenne montrera, en 1982, 78 sorties sur le Saint-Laurent et 5350 passagers. Plus de 100 bénévoles auront été impliqués d'une maniere ou d'une autre dans cet effort sans précédent à la Linnéenne; néanmoins, tous conviennent que nos structures organisationnelles ne conviennent plus pour poursuivre cette expansion. Aussi. une filiale sans but lucratif doit être fondée; elle aura pour nom la Société linnéenne- Saint-Laurent.

La Linnéenne-Saint-Laurent aura pour mandat principal d'organiser des croisières, tandis que la Linnéenne du Québec poursuivra l'ensemble de ses activités dans l'axe du Saint-Laurent mais en se cantonnant à la rive. L'année 1983 commence bien avec la réception, en février, du prix du Gouverneur général du Canada en matière de tourisme et de conservation. Les bonnes nouvelles vont continuer d'arriver à l'une et l'autre sociétés. En effet, à la Linnéenne - Saint-Laurent, nous obtenons une subvention dans le cadre d'un programme fédéral d'aide à l'emploi pour six personnes; nous sommes des lors assurés de poursuivre les croisieres avec encore plus d'intensité. Un autre evénement marquant tient au fait que nous décidons d'organiser des croisières aux baleines à partir de la rive nord (Tadoussac et Baie-Sainte-Catherine) pour une durée de trois heures (25\$) en sus de celles qui partiront de Trois-Pistoles pour une durée de neuf heures (75\$). Le grand succès que nous y connaissons marque pour ainsi dire le début de la fin des croisières à Trois-Pistoles où les organismes et les partenaires ne paraissent guère enclins à collaborer, en dépit du fait que nous y assumons tous les risques. Du côté de la Linnéenne du Québec, nous en venons a une entente avec Parcs Canada afin de gérer la première halte cốtière au Canada. Elle aura pour objectif de sensibiliser les gens à l'intérêt de protéger les bélugas et de faire partager au public notre intérét pour la désignation d'un parc marin national à l'embouchure du fjord du Saguenay. Toutes ces opérations me donnent à penser qu'une Union québécoise pour la conservation de la nature (UQCN) dans laquelle adhéreraient tous les organismes impliqués serait l'outil indispensable pour soutenir à $\mathrm{I}^{\top}$ anné l'intérêt des Québécois que nous éveillons lors des croisières ou de leurs visites a l'Aquarium de Québec.

\section{De nombreux dossiers et activités}

La cinquante-cinquième année de la Société linnéenne du Québec est mouvementée. La Linnéenne est dès lors passée d'une société d'allure régionale à une stature plus provinciale. En 1983-84, elle s'intéresse acti- vement à de nombreux dossiers touchant le fleuve dont:

- un projet de politique sur les pares marins nationaux (Parcs Canada);

- un projet de politique sur la gestion de l'habitat poisson (Péches et Océans);

- le plan d'amenagement du parc national de l'archipel de Mingan (Parcs Canada);

- des objections à la construction de la marina de Lévis et la demande d'une politique sur le littoral;

- I'animation sur les bartures de Beauport et la demande d'une étude récrếotouristique avant l'extension du port de Québec;

- une étude d'impact et une demande d'audiences publiques concernant le projet d'assainissement des caux usées de la Communauté Urbaine de Québec,

- un appuí à la proposition d'un parc national a la Grosse-Ile;

- la promotion du toponyme Archipel de Montmagny;

- la mise sur pied de la première halte côtière au Canada à l'embouchure du Saguenay;

- la poursuite de la campagne de sensibilisation à la protection des bélugas;

- une proposition de déménager le pavillon $\mathrm{H}_{2} \mathrm{O}$ près de l'Aquarium de Québec et d'y recréer un centre d'interprétation sur le Saint-Laurent;

Du côté de la Linnéenne-Saint-Laurent, plusieurs evénements surviennent aussi. Le personnel est maintenant aguerri aux dimensions touristiques et économiques du Saint-Laurent. Il se trouve toutefois quelques ombres au tableau: les subventions gouvernementales fédérales seront très réduites, trop pour une deuxième annee, et la compagnie Via Rail est prête à faire faux bond, malheureuse sur rails et malheureuse de nos succès; il nous faut donc recalculer et augmenter les prix. Les croisières printanières pour voir les grandes oies blanches subissent un certain recul mais nous aurons l'occasion de nous reprendre lors des activités nautiques de Québec 1534-1984. Et nous voilà rendus à $l^{\dagger}$ aube de la saison aux baleines lorsque le propriétaire du Grand Hôtel de Tadoussac exerce des pressions sur notre batelier pour l'embaucher en exclusivité. L'entente est reniée par ce dernier et, pendant plus d'une semaine, la Societé cherche un autre partenaire car sa clientèle est devenue considérable: elle trouve enfin un bateau qui répond adéquatement à ses besoins. C'est à ce moment que la concurrence véritable naît dans ce dossier touristique. En effet, trois organisations modemes existent gráce à nos bons soins a Trois-Pistoles, Tadoussac et BaieSainte-Catherine. La saison sera difficile pour les administrateurs et notre personnel, divisés sur les orientations à prendre dont celle-là même de cesser nos activités. Nous regrettons amèrement de ne pas posséder notre propre bateau et d'avoir accordé une si grande confiance à nos multiples partenaires de soutien. L'ensemble du dossier des croisières est finalement repris par la societé-mère.

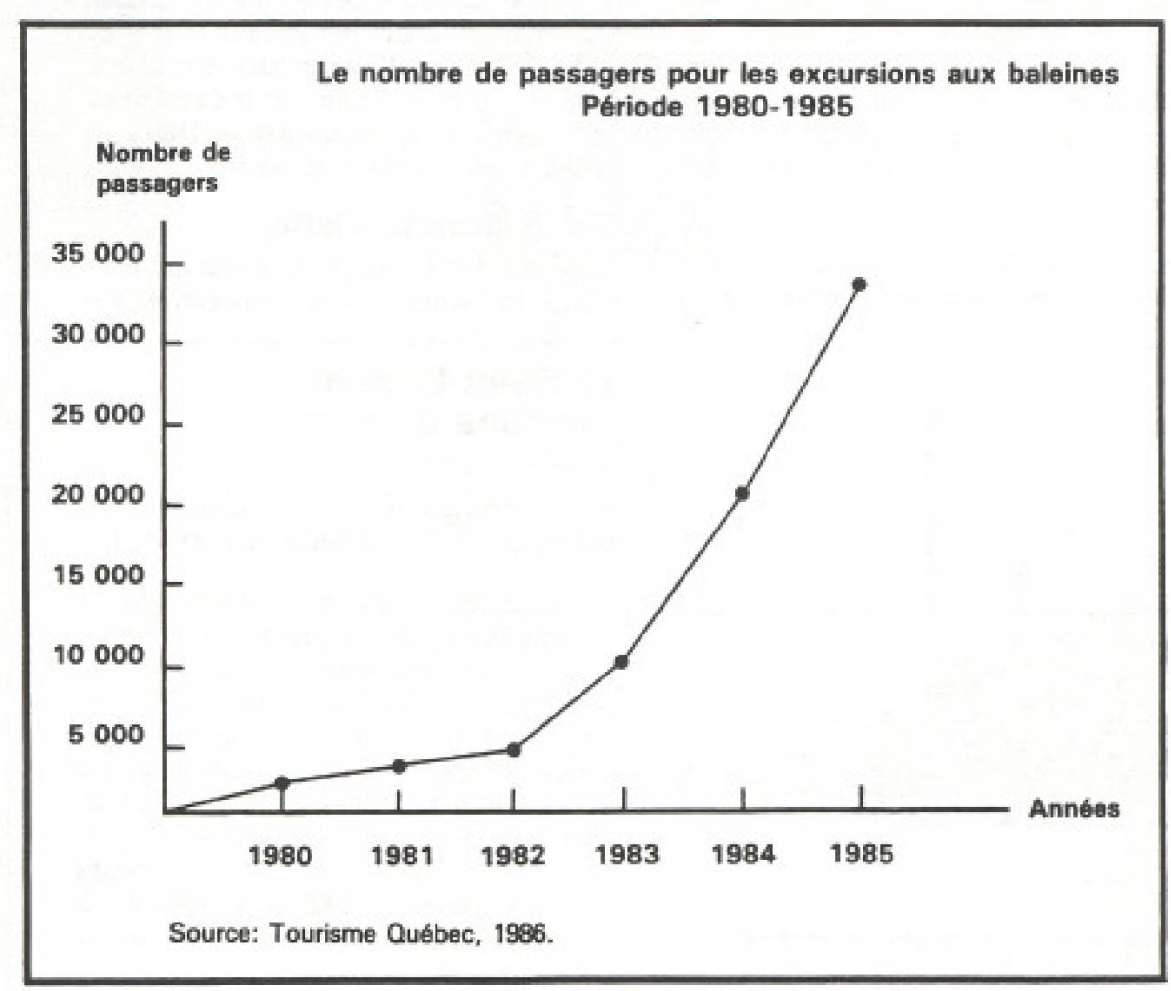




\section{Les risques et les efforts de la linnéenne}

En 1985, la Linnéenne du Québec poursuit son expansion le long des rives du SaintLaurent: après la cogestion à I"A quarium de Québec, la gestion à la halte cötière de pointe Noire, elle soumissionne pour être cogestionnaire à la réserve nationale de faune de Cap-Tourmente qu'elle obtiendra le ler avril 1985 . Le temps qu'il reste aux bénévoles va au dossier des croisières pour en effectuer un bilan. Ainsi, pendant quelques années, la Linnéenne a pris tous les risques de recherche, d'expérimentation et de commercialisation tout en respectant le rốle des bateliers privés; dans un deuxième temps, nous avons partagé les risques avec les bateliers retenus pour un produit touristique donné en réalisant bien que ces derniers tentaient de nous marginaliser, saison après saison. Entre toutes les options examinées, nous en acceptons finalement une nouvelle venant d'un nouveau batelier de Tadoussac qui offre de prendre tous les risques à sa charge en contrepartie d'un support exclusif pour la référence de clientêle et les services d'interprétation. Nous serons donc trois organisations à desservir le public sur lá rive nord pour un marché qui continue à croître: celuì-ci (figure 1) entraine des retombées économiques dans la région de plus de trois millions de dollars.

Le 23 novembre 1985, la Linnéenne reçoit le prix François de B.-Gourdeau, remis annuellement par le ministère du Loisir, de la Chasse et de la Pêche du Québec à un organisme de conservation et elle continue en 1986 sur sa lancée initiale en poursuivant sensiblement les mëmes dossiers terrestres et nautiques. Une année de consolidation où nous n'ajoutons qu'un produit d'importance, soit l'instauration d'une croisière expérimentale comprenant un départ de Tadoussac en voilier et un sêjour à l'île Verte .

Après tant d'efforts, le Saint-Laurent est redevenu un puissant attrait touristique. $f$

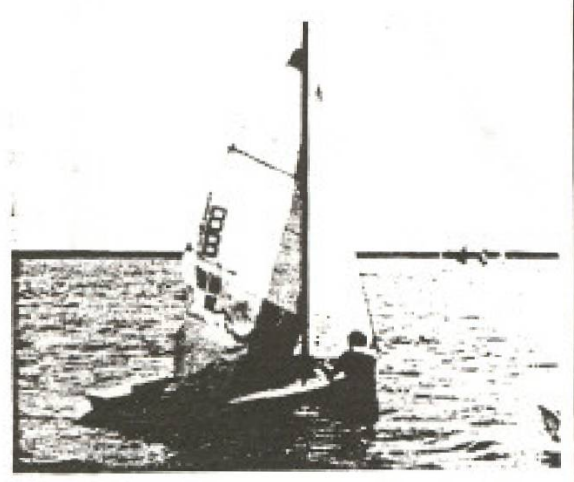

\section{Ribibrence}

(1) Mme Suzanne Nomandeau et M. Rend Roy. furent aussi las autres responsabies du projet.

\section{Le Saint-Laurent} de demain

(suite de la page 25)

voie de faire subir au Vieux-Port. Dans cette ville au cachet européen et historique unique, on opte pour le moderne, les constructions commerciales en hauteur, sans souci d'intégration, sans plan directeur ni consultation publique. Rien, pourtant, ne se démode plus rapidement que le style futuriste!

De Lotbinière à Saint-Jean-Port-Joli, le plan de développement touristique du Paysde-l'Érable propose une série de projets de mise en valeur, notamment à Pointe au Platon sur la côte de Lotbinière, dans le VieuxLévis et le Vieux-Lauzon. Plus en aval, le plan propose de faire de la Côte-du-Sud une zone de destination touristique reposant sur la mise en valeur du parc historique national de Grosse-Ile, le bassin de Montmagny et le reste de l'archipel. Selon Parcs Canada, le projet touristique de Grosse-Ile pourrait générer une affluence de 80000 visiteurs par année.

Dans Charlevoix, le projet de création d'un parc marin à l'embouchure du Saguenay et sur le littoral, la mise en valeur du site de l'ancien chantier naval de Saint-Joseph-dela-Rive, la station de Petite-Rivière-SaintFrançois dont l'intérêt réside en grande partie dans le mariage entre la montagne et le fleuve, sont au nombre des attraits d'avenir.

Les plans de développement touristique des régions du Bas-Saint-Laurent et de la Gaspésie réalisés il $\mathrm{y}$ a quelques années sont en voie de révision. Mentionnons toutefois une étude en cours portant sur la faisabilité d'un centre d'interprétation maritime à Pointe-au-Père. Enfin, sur la Côte-Nord, les régions de Manicouagan et Duplessis préparent leurs plans respectifs.

\section{De la grande visite}

L'industrie et le marché mondial des croisières touristiques sont concentrés aux

\section{Le Saint-Laurent mémoire du pays}

(suite de la page 4)

miers chenaux qui ont favorisé en ses berges ports, industries et urbanisation.

A Verchères et Lavaltrie. Donnaconna et Deschaillons, Kamouraska et Cap-àl'Aigle, des gens regardaient l'autre rive sans jamais y aller, ou attendaient l'hiver; aux Ilets-Caribou et Cap-Chat, à Magpie et Cloridorme, il y a des gens qui ne voient jamais l'autre rive. Mais d'Alençon à SaintJoseph-de-Sorel, de Deschambault à Lotbinière, de Québec à Lévis, des Escoumins à Trois-Pistoles, de Matane à Godbout, i] y a chaque jour le réseau sans cesse tressé des traversiers.
États-Unis. Les grandes compagnies maritimes, considérant le quadrilatère que forme le continent nord-américain, ont d'abord exploité à fond le sud-est, les Caraibes, puis le sud-ouest, la Riviera mexicaine. Ces dernières années, le nordouest. I Alaska, a connu un essor touristique remarquable. Reste le nord-est, constitué de la Nouvelle-Angleterre. des Maritimes et du Saint-Laurent (c'est-ă-dire le lieu des anciennes croisières).

Attendons-nous à voir, au cours des prochaines années, la visite de plus en plus fréquente des magnifiques navires de la nouvelle armada de cette industrie en pleine expansion. A eux seuls, ces navires de grande classe ${ }^{(9)}$ constituent une attraction. $f$

\section{Réfárences et notes}

(1) Document rendu public en mars 1987 par le MLCP $220 \mathrm{p}$

(2) A noter que de tels organismes se sont développés alleurs en fonction de cours d' eau aux dimen. sions plus réduites.

(3) II y en a certes trop peu. Entre Montreal et Québec, cela peut śsexpliquer en partie par le manque đopportunite et d'accessibilite mais plus en aval, le phenomene se justifie moins bien.

(4) A surveiller: les rebsultats de la Conférence internationale des maires du Saint-Launont ot des Grand: Lacs la Quebec les 28 et 29 mai 18d7). Cette conférence bi-nationale a étudié les avenues nouvel. les pour promouwoir le commerce et le tourisme a l'intérieur du systeme maritime SaintLaurent/Grands Lacs.

(5) Le tourisme à Montreal, bilan et perspectives de développement, OPDO, 1986, 267 p.

(6) Marsan, Jean-Claude, Montribal, une esquisse du futur, IORC, 1983.

(7) Association touristique regionale du Coeur du Quebec, Plen de developpement touristique régional du Coeur du Qubbec, septembre 1986.

(8) Ibidem, p. B6

(91) Le Veracruz, gue I'on connait bien, est classe deux 6toiles (classification de Berlitz). A titre de com. paraison, les navires qui depuis peu sillonnent le Saint-Laurent, tels le Royal Viking Sea, le Rova Viking Sky at POceen Princess, sont claseds cing etoiles, te Stella Solaris at is Royal Odvssey, qua tre thoiles. La Sitmar Cruises Line projette de venir a Montréal en 1988. Ses naviras sont clased's quatre étoiles.
D'Anticosti à la plaine, le Saint-Laurent habille le Québec: corsage entrouvert, il laisse, sous des bordures de dentelle, soupçonner des charmes épanouis, imaginer des formes pleines et généreuses, des grains d'une peau tressaillante qui s'appellent I'Islet, Gaspésie, Côte-Nord et Mauricie. Désirer des abandons. Il pare sa gorge d'une riche rivière d'îles emprisonnées et libérées tout à la fois, sculptées, pressées, caressées.

Quand le Saint-Laurent endort ses iles à l'automne, je suis le père Didace qui pousse, du long batton, son bateau entre celles de Sorel. Je vais, derrière cet homme debout, à la rencontre de notre commun ancêtre. $f$ 